\title{
Leaky Gut and Gut-Liver Axis in Liver Cirrhosis: Clinical Studies Update
}

\author{
Hiroshi Fukui \\ Department of Gastroenterology, Nara Medical University, Kashihara, Japan
}

\section{Article Info}

Received January 15, 2020

Revised June 9, 2020

Accepted June 9, 2020

Published online October 21, 2020

\section{Corresponding Author}

Hiroshi Fukui

ORCID https://orcid.org/0000-0003-1832-7338

E-mail hfukki@icloud.com

\begin{abstract}
Portal blood flows into the liver containing the gut microbiome and its products such as endotoxin and bacterial DNA. The cirrhotic liver acts and detoxifies as the initial site of microbial products. In so-called "leaky gut," the increased intestinal permeability for bacteria and their products constitutes an important pathogenetic factor for major complications in patients with liver cirrhosis. Prolonged gastric and small intestinal transit may induce intestinal bacterial overgrowth, a condition in which colonic bacteria translocate into the small gut. Cirrhotic patients further show gut dysbiosis characterized by an overgrowth of potentially pathogenic bacteria and a decrease in autochthonous nonpathogenic bacteria. Pathological bacterial translocation (BT) is a contributing factor in the development of various severe complications. Bile acids (BAs) undergo extensive enterohepatic circulation and play important roles in the gut-liver axis. BT-induced inflammation prevents synthesis of BAs in the liver through inhibition of BA-synthesizing enzyme CYP7A1. A lower abundance of $7 \alpha$-dehydroxylating gut bacteria leads to decreased conversion of primary to secondary BAs. Decreases in total and secondary BAs may play an important role in the gut dysbiosis characterized by a proinflammatory and toxic gut microbiome inducing BT and endotoxemia, as addressed in my previous reviews. Selective intestinal decontamination by the use of various antimicrobial drugs for management of complications has a long history. Lactobacillus GG decreasing endotoxemia is reported to improve the microbiome with beneficial changes in amino acid, vitamin and secondary BA metabolism. Current approaches for hepatic encephalopathy are the use of nonabsorbable antibiotics and disaccharides. Probiotics may become an additional therapeutic option for advanced liver cirrhosis. (Gut Liver 2021;15:666-676)
\end{abstract}

Key Words: Gut-liver axis; Endotoxin; Gut dysbiosis; Gut dysmotility; Liver cirrhosis

\section{INTRODUCTION}

The cirrhotic liver act as the initial site of their detoxification for microbial products from the portal blood. The increased intestinal permeability for bacteria and their products, which is called as leaky gut, is common in liver cirrhosis (LC) and induces an important pathogenetic factor for major complications. Prolonged gut transit induces intestinal bacterial overgrowth, a pathological state in which colonic bacteria translocate into the small intestine. Cirrhotic patients further revealed gut dysbiosis characterized by an overgrowth of potentially pathogenic bacteria and a decrease in autochthonous nonpathogenic bacteria.
Pathological bacterial translocation (BT) is a contributing factor. Bile acids (BAs) undergo extensive enterohepatic circulation. BAs derangement play an important role in the gut dysbiosis characterized by a proinflammatory gut microbiome inducing BT and endotoxemia. Various trial to improve these sequences has been tried for many years. I conducted a PubMed search using search terms including "endotoxin," "gut liver axis," and "liver cirrhosis" between 1980 to 2019. This review is fundamentally based on the conference text which I presented in Seoul International Digestive Disease Symposium 2016 (SIDDS) in 2016. Some recent important manuscripts about leaky gut and gut-liver axis in LC ware also included in the present manuscript. 


\section{MAIN PLAYERS IN THE GUT-LIVER AXIS}

LC is a terminal pathological change in the long history of variable chronic liver diseases characterized by liver fibrosis and the alterations of normal liver architecture into cirrhotic nodules. ${ }^{1}$ Subsequent portal hypertension underlies various clinical complications in patients with LC. ${ }^{1}$ Bacterial infections explain elevated morbidity and mortality ${ }^{2}$ and infections increase mortality four-fold in patients with LC. ${ }^{3}$ Although urinary, respiratory, ascitic fluid infections and bacteremia are well-known infections, spontaneous bacterial peritonitis (SBP) frequently developed in advanced cases.

\section{Endotoxin and other microbial products which disturb the gut-liver axis}

BT or microbial translocation is defined as the migration of viable microorganisms or their products from the gut lumen into the mesenteric lymph nodes and other tissue and organs. ${ }^{4}$ Passage of viable bacteria and their products from the intestinal lumen through the intestinal wall and their translocation is the popular backgrounds for the occurrence of infections such as SBP or bacteremia in LC. ${ }^{5,6}$ Bacterial endotoxin (i.e., lipopolysaccharide, LPS) is a component of the Gram-negative bacterial wall and is important as one of pathogen-associated molecular patterns for Toll-like receptors (TLRs). After the translocation microbial products like LPS activate hepatic Kupffer cells (KCs) through pattern recognition receptors, such as TLRs and nucleotide-binding oligomerization domain (NOD)like receptors. ${ }^{7}$ TLRs recognize not only bacterial structural components but also fungal and viral components, which induce innate immune responses through cytokine and chemokine production in the liver. ${ }^{7-9}$ Hepatocytes, KCs, hepatic stellate cells (HSCs) and endothelial cells respond to bacterial products through TLRs ${ }^{7}$ and enhance proinflammatory and profibrotic reactions via various cytokines. ${ }^{10}$ Early study using limulus amebocyte lysate (LAL) test showed elevated occurrence of systemic endotoxemia in patients with LC. ${ }^{11}$ The LAL test further detected portal venous endotoxemia in $42.9 \%$ patients without liver diseases. ${ }^{11}$ Quantitative endotoxin assays performed thereafter showed elevated systemic endotoxin values with the progression of LC..$^{12-14}$ Close associations of endotoxemia with important complications including hyperdynamic circulation, portal hypertension, renal, pulmonary, cardiac, and coagulation disturbances have been recognized in patients with LC. ${ }^{10}$ Recently an indirect assay of endotoxemia by endotoxin activity assay (EAA) is prevailing. We noticed high EAA results in cirrhotic patients with refractory ascites, jaundice and hepatic encephalopathy (HE) by this method (Fig. 1). They are positively correlated to serum total bilirubin, fibrin degradation product (FDP) and Ddimer levels and negatively correlated to albumin level and prothrombin time. Selective intestinal decontamination by antibiotics has been reported worldwide. ${ }^{15}$

\section{Moderators of the gut liver axis in cirrhotic patients}

\section{1) Short chain fatty acids}

Human gut microbiota acts substrates such as resistant starch and non-starch polysaccharides not completely hydrolyzed by host enzymes in the small gut. ${ }^{16}$ The chief fermentation products are short chain fatty acids (SCFAs) including acetate, propionate, and butyrate. ${ }^{16}$ Butyrate provides an energy source for the colonic epithelium. While acetate and propionate work as substrates for gluconeogenesis and lipogenesis. ${ }^{1718}$ The SCFAs provide an additional energy source for the body, thus constitute 3\% to $9 \%$ of daily caloric intake. ${ }^{19}$ SCFAs exhibit various physiological functions raging from mucoprotection, immune regulation and variable metabolism as well, ${ }^{20,21}$ thus having a direct and indirect effect on human bodies. The main bacteria that produce SCFAs are Eubacterium and Ruminococcaceae. $^{21}$

\section{2) Bile acids}

BAs are hydroxylated C-24 cyclopentanophenanthrene sterols converted from cholesterol in hepatocytes. ${ }^{22}$ Cholesterol $7 \alpha$-hydroxylase (CYP7A1) synthesizes the dihydroxy BA chenodeoxycholic acid (CDCA) and the trihydroxy BA cholic acid (CA) in the hepatocytes. These primary BAs are conjugated with taurine or glycine before being secreted from the liver and stored in the gallbladder as main

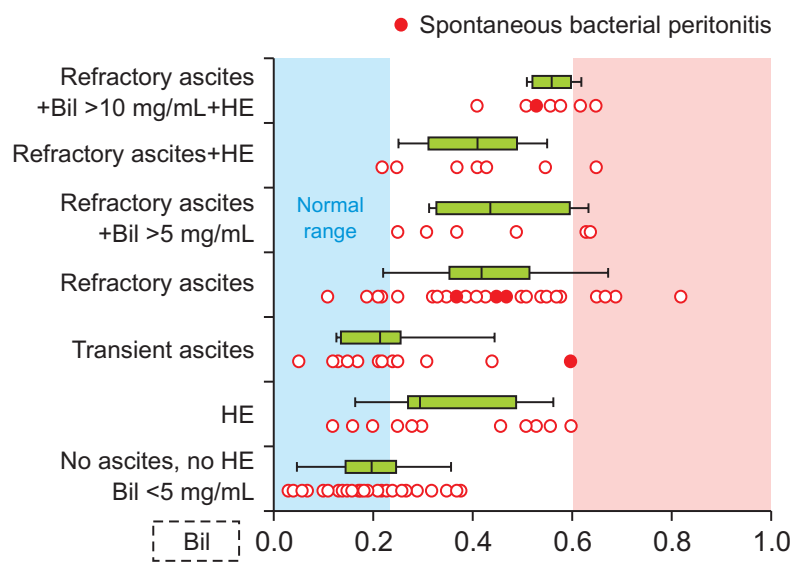

Fig. 1. Blood endotoxin activity in patients with liver cirrhosis and its complications (by EAA). The area with blue color shows normal EAA in healthy subjects.

Bil, serum total bilirubin; $H E$, hepatic encephalopathy; EAA, endotoxin activity assay. Adapted from Fukui H. Diseases 2019;7:58. ${ }^{6}$ 
biliary components. Eating habit stimulates gallbladder contraction and bile secretion into the small gut. ${ }^{23}$ Bile salts can solubilize fats and fat-soluble vitamins and help their uptake. BAs are mostly absorbed in the terminal ileum by the aid of the sodium-dependent BA transporter and flow into the liver through the portal circulation, working as important carrier of portal enterohepatic circulation. The remained BA escapes the enterohepatic circulation and works as substrate for microbial biotransformation in the right colon. ${ }^{22}$ Conjugated primary BAs (as CDCA and CA) undergo microbial modifications including deconjugation, dehydroxylation, hydrogenation to synthesize secondary BAs named as lithocholic acid (LCA) and deoxycholic acid (DCA), respectively. ${ }^{18}$ The colonic $7 \alpha$-dehydroxylating bacteria such as Ruminococcaceae, Lachnospiraceae and Blautia mainly work in this conversion process. BAs are now recognized as signaling molecules which activate specific nuclear farnesoid $\mathrm{X}$ receptor (FXR) and membrane BA-activated G protein-coupled receptor (GP-BAR1) TGR5 in the intestinal wall. ${ }^{24-27}$ Although CDCA is the most potent endogenous FXR ligand. DCA and LCA, can also activate FXR in a smaller dose. TGR5 is activated by nanomolar concentrations of LCA and micromolar concentrations of CA, DCA, and CDCA. ${ }^{28,29}$ By activating various signaling pathways through the binding to FXR in the enterocytes and the parenchymal hepatocytes and to TGR5 in the non-parenchymal hepatocytes, BAs affect various metabolic processes, including cholesterol, triglyceride and glucose metabolism and inflammatory reactions. ${ }^{29} \mathrm{BAs}$ also negatively work on gut bacteria through antimicrobial properties and activation of FXR-induced antimicrobial peptide in the small gut. ${ }^{30}$ Bile inhibits small intestinal bacterial overgrowth (SIBO) and has a trophic effect on the intestinal mucosa, suppresses epithelial internalization of bacteria, works as detergent actions with anti-adherence effects, deactivates endotoxins and gives powerful effects on immune cells in lymphatic tissue in the intestine. ${ }^{31}$ In cirrhotic patients, marked decreases in intestinal intraluminal contents of BAs have been known to increase deconjugation by enteric bacteria. ${ }^{23}$ The defect of bile in the intestine facilitates $\mathrm{BT}^{32,33}$ and enhances susceptibility to bacterial endotoxins. ${ }^{33}$ The transcription factor FXR, a nuclear receptor for conjugated BAs, has now attracted popular attention. Fig. 2 depicts BAs metabolism in the liver and the intestine.

FXR plays a cardinal role in protecting intestinal epithelial integrity and protecting inflammation by depression of nuclear factor kappa-light-chain-enhancer of activated $\mathrm{B}$ cells (NF- $\mathrm{KB})$ signaling and intestinal stimulating antimicrobial peptide release. ${ }^{34,35}$ The FXR agonist obeticholic acid is regarded to improve intestinal antibacterial defense and suppress permeability as well as to decrease gut BT in experimental LC model. ${ }^{36,37}$ In different cirrhotic models it has considered to decrease portal pressure mediated by depressing intrahepatic vascular resistance. ${ }^{37,38}$ Previous human results using obeticholic acid have revealed promising results to improve histological activity and even reduce fibrosis in different liver disturbance, suppressing the gutliver axis. ${ }^{6}$ BAs are in these ways regarded as a mediator to adjust gut-liver axis.

\section{GUT DYSFUNCTION, DYSBIOSIS AND LEAKY GUT IN LC}

Leaky gut is an essential common word that indicates increased intestinal permeability in various human diseases. Most researchers reported small gut dysmotility in

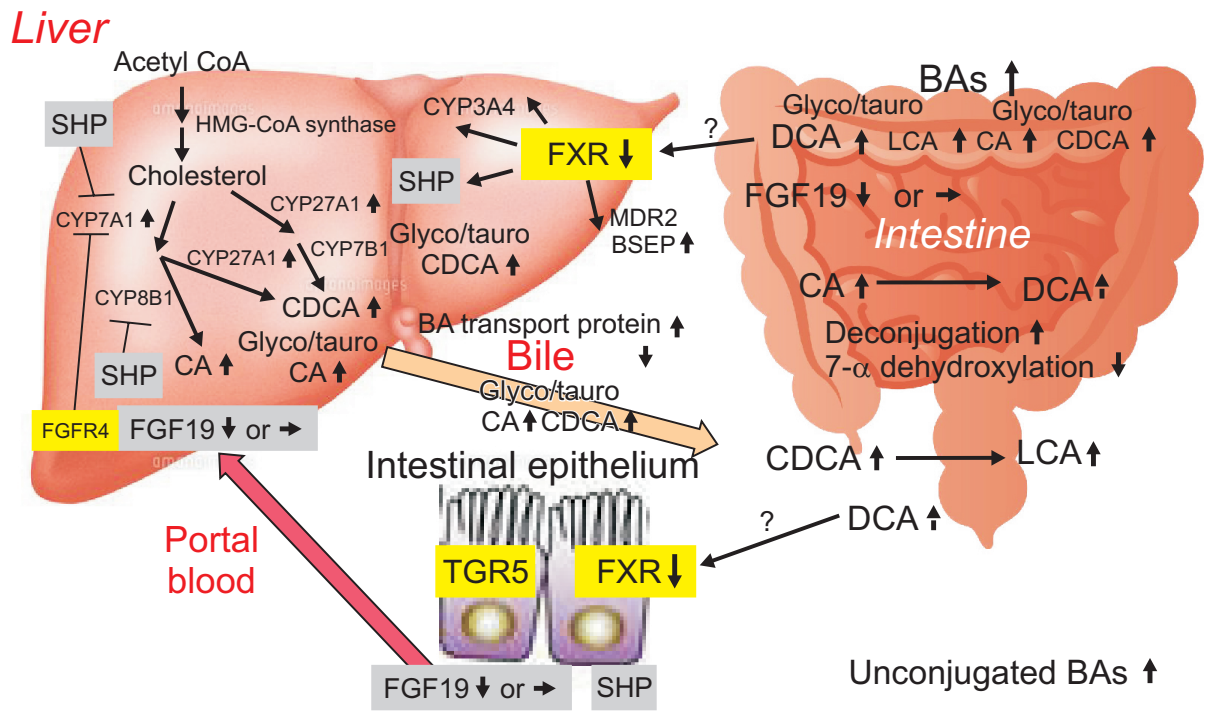

Fig. 2. BA metabolism in the liver and the intestine.

BAs, bile acids; BSEP, bile salt export pump; CA, cholic acid; CDCA, chenodeoxycholic acid; DCA, deoxycholic acid; FGF19, fibroblast growth factor 19; FGR4, fibroblast growth factor receptor 4; FXR, farnesoid $X$ receptor; HMG, 3-hydroxy-3-methylglutaryl; TGR5, G-protein-coupled bile acid receptor; LCA, lithocholic acid; SHP, small heterodimer partner. Adapted from Fukui H. Diseases 2019;7:58. ${ }^{6}$ 
Table 1. Changes in Intestinal Microbiota in Liver Cirrhosis

\begin{tabular}{|c|c|c|c|c|}
\hline Phylum & Class & Order & Family & Genus (species) \\
\hline \multirow[t]{16}{*}{ Firmicutes $\uparrow$} & Bacilli $\uparrow$ & Bacillales & Staphylococcaceae & Staphylococcus \\
\hline & & Lactobacilales & Lactobacillaceae & \\
\hline & & & Streptococcaceae & Lactobacillus L. \\
\hline & & & Enterococcaceae & Streptococcus \\
\hline & Clostridia & Clostridiales & Clostridiaceae & Enterococcus \\
\hline & & & Eubacteriaceae & Clostridium $\downarrow \uparrow$, Clostridium XI $\uparrow$ \\
\hline & & & Ruminococcaceae $\downarrow$ & Eubacterium $\downarrow$ \\
\hline & & & & Subdoligranulum $\downarrow$ \\
\hline & & & & Faecalibacterium $\downarrow$ \\
\hline & & & & Ruminococcus \\
\hline & & & Lachnospiraceae $\downarrow$ & Bacterium $\downarrow$ \\
\hline & & & & Dorea $\downarrow$ \\
\hline & & & & Blautia $\downarrow$ \\
\hline & Negativicutes $\uparrow$ & Selenomonadales & Veillonellaceae $\uparrow$ & Butyrivibrio (B. crossotus $\downarrow)$ \\
\hline & & & Acidaminococcaceae & Veillonella $\uparrow$ \\
\hline & & & & Acidaminococcus $\uparrow$ \\
\hline Actinobacteria $\uparrow$ & Actinobacteria & Bifidobacteriales & Bifidobacteriaceae & Phascolarctobacterium $\downarrow$ \\
\hline Fusobacteria $\uparrow$ & Fusobacteria & Fusobacteriales & Fusobacteriaceae $\uparrow$ & Bifidobacterium $\downarrow$ \\
\hline \multirow[t]{8}{*}{ Bacteroidetes $\downarrow$} & Bacteroidia & Bacteroidales & Bacteroidaceae $\downarrow$ & \\
\hline & & & Prevotellaceae & Bacteroides $\downarrow$ \\
\hline & & & & Prevotella $\uparrow \downarrow$ \\
\hline & & & Rikenellaceae $\downarrow$ & Paraprevotella $\downarrow($ P. xylaniphila $\downarrow)$ \\
\hline & & & Porphyromonadaceae & Alistipes $\downarrow$ \\
\hline & & & & Barnecialla $\downarrow$ \\
\hline & & & & Odoribacter $\downarrow$ \\
\hline & & & & Parabacteroides (P. distasonis $\downarrow$ ) \\
\hline \multirow[t]{7}{*}{ Proteobacteria $\uparrow$} & $\beta$-Proteobacteria & Burkholderiales & Alcaligenaceae $\uparrow$ & Tannerella $\downarrow$ \\
\hline & & & Burkholderiaceae & \\
\hline & & & Ralstoniaceae & Burkholderia $\uparrow$ \\
\hline & $\gamma$-Proteobacteria $\uparrow$ & Enterobacteriales & Enterobacteriaceae $\uparrow$ & Ralstonia $\uparrow$ \\
\hline & & & & Proteus $\uparrow$ \\
\hline & & & Pasteurellaceae $\uparrow$ & Escherichia $\uparrow$ (E. coli $\uparrow$ ) \\
\hline & $\delta$-Proteobacteria & Desulfovibrionales & Desulfovibrionaceae & \\
\hline
\end{tabular}

Adapted from Fukui H. Diseases 2019;7:58.

cirrhotic patients. Marked changes in the gut contraction pattern were reported in the previous manometric researches. The orocecal transit time (OCTT), particularly in the small intestine, was observed to be prolonged, which was associated with the grade of LC, the development of SIBO and HE in addition to a preceding history of SBP. Bacteriologically, SIBO determined by proximal jejunal aspirates was observed to be present in about $60 \%$ of patients with LC and is related to endotoxemia. ${ }^{39}$ Delayed small bowel transit was reported in cirrhotic patients accompanied with SIBO, which was related to the abdominal pain and diarrhea. Together with autonomic neuropathy, metabolic derangement including diabetes mellitus, SIBO possibly prolong intestinal transit in patients with LC. Several studies have reported that the gut microbiota is changed in patients with LC especially those with HE (Table 1). A quantitative alteration in Bacteroides/Firmicutes ratio, with an increase in potentially pathogenic bacteria including
Enterobacteriaceae together with a reduction in specific autochthonous commensals were observed. ${ }^{39}$ Bajaj et al. ${ }^{40}$ proposed cirrhosis dysbiosis ratio (CDR), which means the ratio of the amounts of beneficial autochthonous taxa including Lachnospiraceae, Ruminococcaceae, Veillonellaceae and Clostridialesncertae Sedis XIV to those of potentially pathogenic taxa including Enterobacteriaceae and Bacteroidaceae. ${ }^{40}$ This CDR was reported to be negatively correlated to the Model for End-Stage Liver Disease score and the blood endotoxin level in advanced LC. ${ }^{40} \mathrm{~A}$ low CDR was noted to predict an early development of organ failure and death. ${ }^{40}$ The gut dysbiosis in LC has been acknowledged in the previous literatures. ${ }^{6,15,39}$ Structural and functional changes in the intestinal mucosa that enhanced intestinal permeability for bacteria and their products, which have been noted in patients with LC. They are chief pathogenetic factors for several grave complications. The etiology of intestinal barrier dysfunction in LC is probably 
multifactorial, including alcohol taking, portal hypertension, SIBO, local infection, endotoxemia, immunological disturbances and continuous medications. The question of whether this intestinal barrier dysfunction estimated by the $\mathrm{CDR}$ is accompanied by functional or structural changes in the epithelial tight junction proteins is unresolved. Disruption of the intestinal barrier induces the passage of bacteria and their products from the intestinal lumen, thereby allowing potent inflammatory reactions, such as various infections and affecting portal and systemic circulatory disturbances. Gut dysbiosis, intestinal dysfunction and endotoxemia are key contributors in these processes. All of them induce inflammation in the liver and multiple extrahepatic organs and tissues, enhancing the progression of LC and its complications.

Madrid et al. ${ }^{41}$ studied bowel motility using perfused catheters with external transducers in cirrhotic patients and these affect the migrating motor complex. Absence of cycling activity was often noted in advanced cirrhotic patients with Child-Pugh $C$ grades. In these severe cases, increased amplitudes and frequency of clustered contractions were observed. The authors considered that these findings are probably associated with the prolonged transit time. ${ }^{41}$ Recordings of antroduodenojejunal pressure showed that prolonged clusters were often observed in cirrhotic patients who had portal hypertension. ${ }^{41}$ Combined with the above results from bacterial cultures of jejunal samples, they have speculated that high portal pressure may be associated with the small intestinal dysfunction in cirrhotic patients. ${ }^{41}$

The OCTT measured by a lactulose load was prolonged in cirrhotic patients with concomitant HE. ${ }^{42}$ The OCTT as determined by a scintigraphic technique was longer in advanced cirrhotic patients who are waiting liver transplantation. ${ }^{43}$ Radiologic procedure showed that $38 \%$ of cirrhotic patients had longer small intestinal transit which was associated with abdominal pain and diarrhea. ${ }^{44} \mathrm{~A}$ later study by means of a wireless motility capsule (Smart-Pill; Medtronic, Minneapolis, MN, USA) by Chander Roland et al..$^{45}$ also showed that decompensated cirrhotic patients have slower intestinal transit times compared with compensated cirrhotic patients. A noninvasive hydrogen breath test revealed that the OCTT was prolonged in cirrhotic patients related to hepatitis B virus infection. ${ }^{46}$ They further reported that patients with hepatocellular carcinoma and viral LC revealed delayed gastrointestinal transit. ${ }^{46}$ Small intestinal dysmotility was more remarkable in cirrhotic patients with a history of SBP. ${ }^{47}$ Another study ${ }^{48}$ showed delayed OCTT in patients with nonalcoholic LC. The primary role of prolonged small intestinal transit in BT of patients with LC can be expected from a pilot trial showing that it precedes the appearance of bacterial DNA in serum and ascites. ${ }^{49}$ Total and left colonic transit times were shorter in cirrhotic patients with accelerated colonic transit is a pathogenetic factor for diarrhea. A magnetbased motility tracking system disclosed that cirrhotic patients with portal hypertension showed faster transit in the proximal small intestine. ${ }^{50}$ Transmucosal passage of microbiota across the intestine is regarded as an important factor for $\mathrm{BT}^{51}$ The gut epithelium plays an important role in immune homeostasis as the first barrier against BT. ${ }^{52,53}$ The gut barrier system of intestinal epithelial cells prevents BT. ${ }^{7}$ The intestinal barrier is constituted mainly by intestinal epithelial cells and their mucinous components. ${ }^{5}$ Intercellular junctions including tight junctions and gap junctions persuade a selective passage of substances. ${ }^{5}$ Structural and functional changes in the intestinal mucosa increasing BT are often observed. ${ }^{5}$ Portal hypertensive gastroduodenopathy defined by enlarged mucosal and submucosal vessels were observed in cirrhotic patients with mild or no inflammatory infiltrate and epithelial erosion. ${ }^{54}$ This condition is related to increased susceptibility to injury from noxious factors reflected in an increased prevalence of peptic ulcer in these cirrhotic patients. ${ }^{55}$ The cause of mucosal damage probably include a decrease in potential differences related to gastric mucosa ${ }^{56}$ and disturbed bicarbonate secretion. ${ }^{57,58}$ Patients with primary biliary cholangitis sometimes revealed increased permeability as well, ${ }^{59}$ although no structural alterations in the intestinal mucosa have been reported. Further debate concerning the intestinal dysfunction in LC were summarized in the previous review. ${ }^{6,39}$ Nonvascular changes such as augmented apoptosis, fibromuscular proliferation, enhanced intraepithelial lymphocytes and shortened and atrophic villi with decreased villous-crypt ratio have been reported in LC. ${ }^{60,61}$ Some of these changes are ascribed to changes in brush border enzymes as well as cell and membrane enzymes. ${ }^{62}$ The capsule endoscopy enabled us to evaluate mucosal alterations in the small intestine with portal hypertensive enteropathy. These changes were inflammation-like abnormalities (edema, granularity, friability and erythema) as well as vascular lesions. ${ }^{63}$ Portal hypertensive enteropathy was noted more than $60 \%$ of cirrhotic patients with chronic anemia and a history of variceal bleeding. ${ }^{64}$ The macroscopic change suggesting edema may be mediated by a rise in interstitial hydration due to marked elevation in intestinal capillary filtration in cirrhotic patients with portal hypertension. In case of chronic advanced portal hypertension, the intestinal fluid content was elevated by up to $40 \%{ }^{65}$ Intestinal barrier dysfunction has been regarded as an important pathogenetic factor of several complications in LC. ${ }^{66}$ Portal hypertension, alterations in the intestinal microbiota, inflammation and 
oxidative stress can influence the barrier function of both the small and the large intestine and probably result in the occurrence of cirrhotic complications. ${ }^{28}$

There has been a long-lasting discussion about the pathological role of enhanced intestinal permeability in cirrhotic patients. ${ }^{67}$ An Italian study reported that intestinal hyperpermeability was more general in cirrhotic patients with a preceding $\mathrm{SBP}^{68} \mathrm{~A}$ Korean study insisted that it was a predictor of bacterial infections. ${ }^{69}$ Three studies ${ }^{63,67,68}$ reported a higher intestinal permeability in patients with LC and ascites, although other three studies did not report a significant difference. ${ }^{69-71}$ Contrasting data have been reported on the relationship between HE and intestinal permeability. ${ }^{28}$ Methodological problems exist when interpreting these conflicting data. ${ }^{72,73}$ Some authors used sugars ${ }^{68,74,75}$ whereas others used more reliable isotope probes. ${ }^{68,69,71}$

Mucosal intestinal permeability by urinary excretion of orally taken nonmetabolizable sugars gave the researchers some information about discrimination between paracellular and transcellular fluxes. ${ }^{76}$ The probes seems to traverse the epithelium in one of three ways: paracellular, transcellular aqueous or transcellular lipid. ${ }^{77}$ Villous tight junctions, reflecting the transcellular pathway, are more accessible to intestinal compounds and more selective for smaller compounds compared with crypt tight junctions. ${ }^{77}$ Monosaccharides including mannitol are absorbed through this transcellular pathway and reflect the grade of absorption of small molecules. Disaccharides (i.e., lactulose and mannose) are absorbed through the paracellular junction complex such as tight junctions and extrusion zones of the intervillous spaces reflecting the permeability of larger molecules. ${ }^{75,78}$ The urinary ratio of two different probes has been used as an accurate indicator of intestinal permeability, on the bases that the premucosal and postmucosal factors affect the probes equally and the urinary excretion ratio should not be influenced by the above factors. ${ }^{77,79,80}$ The lactulose/mannitol ratio (LMR) may express an index to evaluate intestinal permeability, and its increase has been regarded as a marker of hyperpermeability. ${ }^{28,81}$ In most studies, this ratio was elevated in patients with LC, ${ }^{28}$ especially those with advanced LC. ${ }^{68,74}$ Alcoholic liver disease also had marked elevations in lactulose excretion with an elevated LMR. ${ }^{81} \mathrm{~A}$ report from Pascual et al. ${ }^{74}$ described a higher lactulose excretion together with a comparable mannitol excretion in patients with LC. Pijls et al. ${ }^{82}$ noted that small intestinal permeability measured by the lactulose/rhamnose ratio is not changed, whereas large intestinal permeability is increased in patients with compensated LC of mixed etiology, although they could not deny a tendency of increased small intestinal permeability in alco- holic cirrhosis. As a larger number and increased diversity of microbiome in the large intestine, higher permeability of this site probably elevated risk of $\mathrm{BT}^{82}$ Parlesak et al. ${ }^{83}$ reported that the permeability measured by polyethylene glycol (PEG) disclosing high molecular masses (PEG 1,500 and 4,000) was elevated in cirrhotic patients ascribed to alcoholic drinking. They thought PEG as a reliable probe for the measurement of endotoxin translocation on the bases that its homogenous chemical character, adequately adaptable molecular mass and linear, chain-like figure mimicking endotoxin itself..$^{83} \mathrm{~A}$ study by Lee et al. ${ }^{84} \mathrm{de}$ scribed that intestinal permeability estimated by PEG 400 and 3,500 was elevated in patients with LC and ascites. In addition, they noted a higher permeability in patients with advanced LC showing Child-Pugh grade class C. ${ }^{84}$ Kim et al. ${ }^{85}$ in Korea wrote that the intestinal permeability index, the percentage of permeability of PEG 3,350 to that of PEG 400 , was elevated in cirrhotic patients with gastrointestinal bleeding and infections.

\section{SMALL INTESTINAL BACTERIAL OVERGROWTH}

SIBO is a pathological state in which colonic bacteria translocate into the small gut attributable to impaired microvillus function, causing a breakdown of intestinal motility and gut homeostasis. ${ }^{86,87}$ Gastric acid, intestinal peristalsis, intestinal mucosal immunity and biliopancreatic juice inhibit the occurrence of SIBO in healthy subjects. Abnormalities of these factors can induce SIBO ${ }^{88}$ SIBO, which means more than 105 total colony-forming units per milliliter of proximal jejunal contents, has been noted to be present in as many as $59 \%$ of cirrhotic patients. It is related to endotoxin in the blood ${ }^{89}$ SIBO was measured by the breath hydrogen test. SIBO diagnosed with this technique is prominent in cirrhotic patients, especially in those with severe liver dysfunction, ascites and associated $\mathrm{SBP}^{47,90}$ In a study evaluating SIBO using the quantitative cultures of jejunal aspirates, it did not related to the presence of SIBO in patients with LC. ${ }^{5}$ Disturbances in the small bowel manometry and delay in the gut transit is probably associated with the development of SIBO. ${ }^{66}$ The OCTT and small intestinal residence time were prolonged in the patients who had SIBO compared with the patients who had no SIBO ${ }^{88,90}$ Enhancement of orocecal transit by taking cisapride is associated with the inhibition of bacterial overgrowth in most patients with LC and bacterial overgrowth. ${ }^{5}$ Prolonged small intestinal transit in cirrhotic patients is expected to enhance the occurrence of SIBO, which may induce abdominal pain and diarrhea. ${ }^{66}$ 


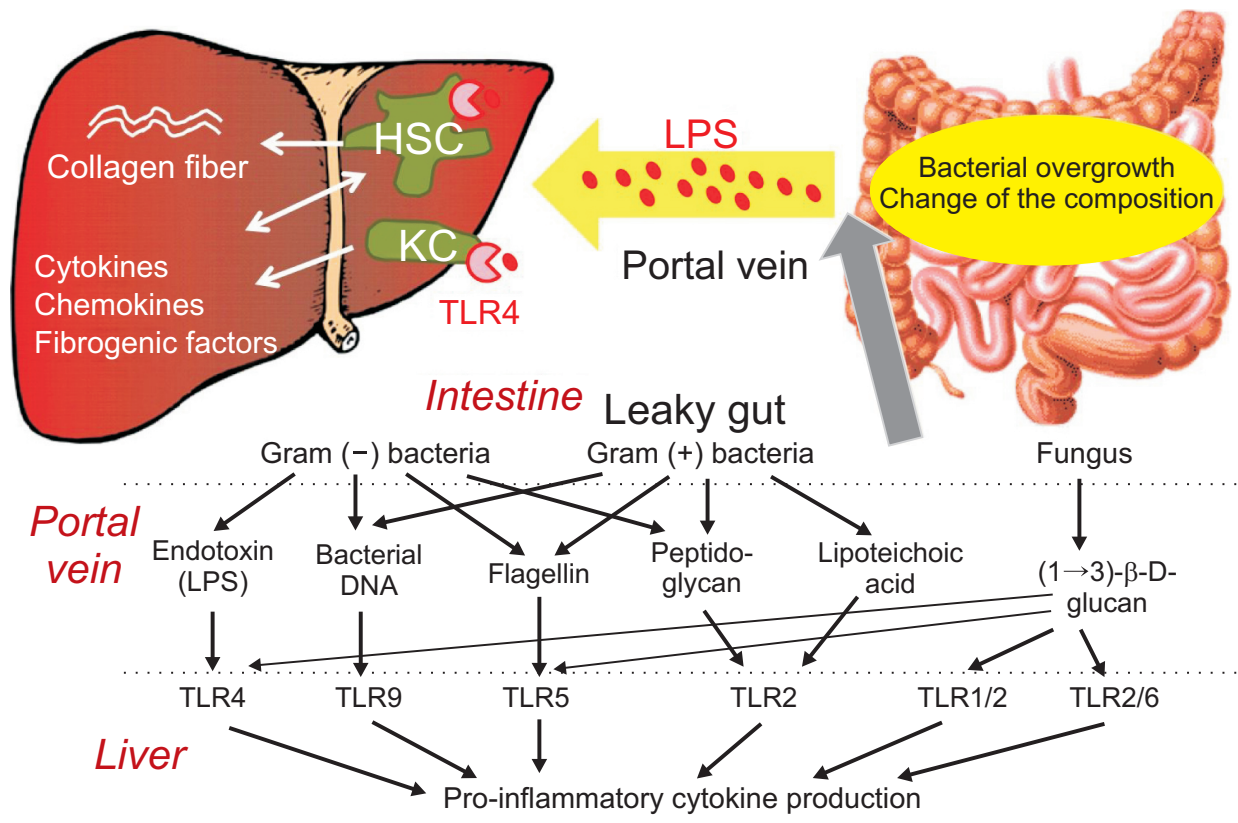

Fig. 3. Gut-liver axis in patients with liver cirrhosis. Translocated bacteria and their products reach the liver via portal circulation and affect near and far extrahepatic organs and general systems in patients with liver cirrhosis. Endotoxin is a representative player in the process. All of the bacterial products stimulate immune responses, causing hepatic proinflammatory cytokine production. Nonimmune cells, such as hepatocytes, HSCs and sinusoidal endothelial cells, respond to bacterial products through TLRs as well. HSC, hepatic stellate cell; KC, Kupffer cell; TLR, Toll-like receptor; LPS, lipopolysaccharide.
The cause of delayed intestinal transit in patients with LC is probably multifactorial. ${ }^{88}$ It could be caused by complications of autonomic neuropathy, metabolic derangements including hyperglycemic state. SIBO itself may provoke delayed intestinal transit. ${ }^{88}$ Antibiotics can shorten the OCTT, which suggests that bacterial overgrowth per se may induce small gut dysmotility. ${ }^{42}$

The microbiota exerts variable functions including salvaging energy, providing vitamins, inhibiting access for pathogens as well as adjusting immunity. ${ }^{91}$ Several studies have showed that the gut microbiota is changed in cirrhotic patients and especially in those who had HE. ${ }^{92}$ Cultureindependent pyrosequencing of stool enables researchers to recognize decline in microbial diversity and characteristic dysbiosis in LC. ${ }^{47,93}$ A quantitative alteration includes the ratio of Bacteroides/Firmicutes, with an increase in pathogenic bacteria such as Enterobacteriaceae $e^{93,94}$ and a reduction of specific commensals (e.g., Lachnospiraceae). ${ }^{94}$ Liu et al. ${ }^{95}$ reported that the overgrowth of potentially pathogenic Staphylococcus spp. and Escherichia coli in the intestine of their patients who had viral LC and minimal HE. Another study from China reported that most patientenriched species were buccal origin, which suggests an invasion of the mouth flora in the stool of cirrhotic patients. ${ }^{96}$ Almost half of the enteral bacteria detected in these patients originated from the oropharyngeal regions than those with their absence in healthy subjects. This underlines the concept of deficient intestinal antimicrobial capacity in patients with LC. The above mentioned U.S. study ${ }^{94}$ showed that cirrhotic patients with HE had augmented Enterobacteriaceae and Alcaligenaceae compared with control subjects and cirrhotic patients without HE. ${ }^{50}$
Another U.S. study reports the clinical meaning of the mucosa-related flora in cirrhotic patients having HE. ${ }^{97}$ The mucosal microbiome was different, with elevated Enterococcus, Veillonella, Megasphaera and Burkholderia and lowered decreased Roseburia abundance in those having $\mathrm{HE}$, although there was no difference in stool microbiota between these cirrhotic patients having HE and those not having HE. ${ }^{94}$ The possible method to adjust gut-liver axis in LC is depicted in Fig. 3. Translocated bacteria and their products reach the liver via portal venous blood and affect liver itself and distant organs in patients with LC. Endotoxin is the most described contributor in these processes. All of the bacterial products enhance immune responses causing proinflammatory cytokine and chemokine production in the liver. Nonimmune cells, such as HSCs and sinusoidal endothelial cells, also respond to bacterial products through TLRs. HSCs are activated through TLR4 and enhance hepatic fibrogenesis as well.

\section{CONCLUSIONS}

Selective intestinal decontamination by the use of various antimicrobial drugs for management of complications has long been tried in patients with LC. Different probiotics has been reported to improve gut dysbiosis and endotoxemia. The trials should be further refined combined with beneficial metabolic changes. Further approaches by antibiotics together with probiotics and prebiotics should be evaluated for the patients with advanced LC, concomitant infection and HE. 


\section{CONFLICTS OF INTEREST}

No potential conflict of interest relevant to this article was reported.

\section{ACKNOWLEDGEMENTS}

The figure 1 was prepared by Dr. Masao Fujimoto in the Department of Gastroenterology, Nara Medical University. The author would appreciate him and other researchers of this department, Nara Medical University, Japan.

\section{ORCID}

Hiroshi Fukui https://orcid.org/0000-0003-1832-7338

\section{REFERENCES}

1. Pinzani M, Rosselli M, Zuckermann M. Liver cirrhosis. Best Pract Res Clin Gastroenterol 2011;25:281-290.

2. Tandon P, Garcia-Tsao G. Bacterial infections, sepsis, and multiorgan failure in cirrhosis. Semin Liver Dis 2008;28:2642.

3. Arvaniti V, D’Amico G, Fede G, et al. Infections in patients with cirrhosis increase mortality four-fold and should be used in determining prognosis. Gastroenterology 2010;139:1246-1256.

4. Pinzone MR, Celesia BM, Di Rosa M, Cacopardo B, Nunnari G. Microbial translocation in chronic liver diseases. Int J Microbiol 2012;2012:694629.

5. Bellot P, Francés R, Such J. Pathological bacterial translocation in cirrhosis: pathophysiology, diagnosis and clinical implications. Liver Int 2013;33:31-39.

6. Fukui H. Role of gut dysbiosis in liver diseases: what have we learned so far? Diseases 2019;7:58.

7. Seo YS, Shah VH. The role of gut-liver axis in the pathogenesis of liver cirrhosis and portal hypertension. Clin Mol Hepatol 2012;18:337-346.

8. Guo J, Friedman SL. Toll-like receptor 4 signaling in liver injury and hepatic fibrogenesis. Fibrogenesis Tissue Repair 2010;3:21.

9. Seki E, Schnabl B. Role of innate immunity and the microbiota in liver fibrosis: crosstalk between the liver and gut. J Physiol 2012;590:447-458.

10. Fukui H. Gut-liver axis in liver cirrhosis: how to manage leaky gut and endotoxemia. World J Hepatol 2015;7:425-442.

11. Prytz H, Holst-Christensen J, Korner B, Liehr H. Portal venous and systemic endotoxaemia in patients without liver disease and systemic endotoxaemia in patients with cirrhosis. Scand J Gastroenterol 1976;11:857-863.

12. Fukui H, Tsujita S, Matsumoto M, et al. Endotoxemia in chronic hepatitis and cirrhosis: epiphenomenon or of pathological relevance? In: Blum H, Bode C, Bode JC, Sartor RB, eds. Gut and the liver. Dordrecht: Kluwer Academic Publishers, 1998:251-262.

13. Lin RS, Lee FY, Lee SD, et al. Endotoxemia in patients with chronic liver diseases: relationship to severity of liver diseases, presence of esophageal varices, and hyperdynamic circulation. J Hepatol 1995;22:165-172.

14. Fujimoto M, Uemura M, Nakatani Y, et al. Plasma endotoxin and serum cytokine levels in patients with alcoholic hepatitis: relation to severity of liver disturbance. Alcohol Clin Exp Res 2000;24(4 Suppl):48S-54S.

15. Fukui H. Gut microbiome-based therapeutics in liver cirrhosis: basic consideration for the next step. J Clin Transl Hepatol 2017;5:249-260.

16. Topping DL, Clifton PM. Short-chain fatty acids and human colonic function: roles of resistant starch and nonstarch polysaccharides. Physiol Rev 2001;81:1031-1064.

17. Leung C, Rivera L, Furness JB, Angus PW. The role of the gut microbiota in NAFLD. Nat Rev Gastroenterol Hepatol 2016;13:412-425.

18. Shen TD, Pyrsopoulos N, Rustgi VK. Microbiota and the liver. Liver Transpl 2018;24:539-550.

19. Schwiertz A, Taras D, Schäfer K, et al. Microbiota and SCFA in lean and overweight healthy subjects. Obesity (Silver Spring) 2010;18:190-195.

20. Willemsen LE, Koetsier MA, van Deventer SJ, van Tol EA. Short chain fatty acids stimulate epithelial mucin 2 expression through differential effects on prostaglandin $\mathrm{E}(1)$ and $\mathrm{E}(2)$ production by intestinal myofibroblasts. Gut 2003;52:1442-1447.

21. Ohira H, Tsutsui W, Fujioka Y. Are short chain fatty acids in gut microbiota defensive players for inflammation and atherosclerosis? J Atheroscler Thromb 2017;24:660-672.

22. Ridlon JM, Kang DJ, Hylemon PB. Bile salt biotransformations by human intestinal bacteria. J Lipid Res 2006;47:241259.

23. Ridlon JM, Alves JM, Hylemon PB, Bajaj JS. Cirrhosis, bile acids and gut microbiota: unraveling a complex relationship. Gut Microbes 2013;4:382-387.

24. Wang H, Chen J, Hollister K, Sowers LC, Forman BM. Endogenous bile acids are ligands for the nuclear receptor FXR/ BAR. Mol Cell 1999;3:543-553.

25. Maruyama T, Miyamoto Y, Nakamura T, et al. Identification of membrane-type receptor for bile acids (M-BAR). Biochem Biophys Res Commun 2002;298:714-719.

26. Keitel V, Häussinger D. Perspective: TGR5 (Gpbar-1) in liver physiology and disease. Clin Res Hepatol Gastroenterol 
2012;36:412-419.

27. Matsubara T, Li F, Gonzalez FJ. FXR signaling in the enterohepatic system. Mol Cell Endocrinol 2013;368:17-29.

28. Pijls KE, Jonkers DM, Elamin EE, Masclee AA, Koek GH. Intestinal epithelial barrier function in liver cirrhosis: an extensive review of the literature. Liver Int 2013;33:1457-1469.

29. Stanimirov B, Stankov K, Mikov M. Bile acid signaling through farnesoid $\mathrm{X}$ and TGR5 receptors in hepatobiliary and intestinal diseases. Hepatobiliary Pancreat Dis Int 2015;14:18-33.

30. Ridlon JM, Kang DJ, Hylemon PB, Bajaj JS. Gut microbiota, cirrhosis, and alcohol regulate bile acid metabolism in the gut. Dig Dis 2015;33:338-345.

31. Hofmann AF, Eckmann L. How bile acids confer gut mucosal protection against bacteria. Proc Natl Acad Sci U S A 2006;103:4333-4334.

32. Parks RW, Clements WD, Smye MG, Pope C, Rowlands BJ, Diamond T. Intestinal barrier dysfunction in clinical and experimental obstructive jaundice and its reversal by internal biliary drainage. Br J Surg 1996;83:1345-1349.

33. Reynolds JV, Murchan P, Leonard N, Clarke P, Keane FB, Tanner WA. Gut barrier failure in experimental obstructive jaundice. J Surg Res 1996;62:11-16.

34. Inagaki T, Moschetta A, Lee YK, et al. Regulation of antibacterial defense in the small intestine by the nuclear bile acid receptor. Proc Natl Acad Sci U S A 2006;103:3920-3925.

35. Gadaleta RM, van Erpecum KJ, Oldenburg B, et al. Farnesoid $\mathrm{X}$ receptor activation inhibits inflammation and preserves the intestinal barrier in inflammatory bowel disease. Gut 2011;60:463-472.

36. Úbeda M, Lario M, Muñoz L, et al. Obeticholic acid reduces bacterial translocation and inhibits intestinal inflammation in cirrhotic rats. J Hepatol 2016;64:1049-1057.

37. Verbeke L, Farre R, Verbinnen B, et al. The FXR agonist obeticholic acid prevents gut barrier dysfunction and bacterial translocation in cholestatic rats. Am J Pathol 2015;185:409-419.

38. Verbeke L, Farre R, Trebicka J, et al. Obeticholic acid, a farnesoid $\mathrm{X}$ receptor agonist, improves portal hypertension by two distinct pathways in cirrhotic rats. Hepatology 2014;59:2286-2298.

39. Fukui H. Gut microbiota and host reaction in liver diseases. Microorganisms 2015;3:759-791.

40. Bajaj JS, Heuman DM, Hylemon PB, et al. Altered profile of human gut microbiome is associated with cirrhosis and its complications. J Hepatol 2014;60:940-947.

41. Madrid AM, Cumsille F, Defilippi C. Altered small bowel motility in patients with liver cirrhosis depends on severity of liver disease. Dig Dis Sci 1997;42:738-742.

42. Van Thiel DH, Fagiuoli S, Wright HI, Chien MC, Gavaler JS. Gastrointestinal transit in cirrhotic patients: effect of hepatic encephalopathy and its treatment. Hepatology 1994;19:6771.

43. Galati JS, Holdeman KP, Bottjen PL, Quigley EM. Gastric emptying and orocecal transit in portal hypertension and end-stage chronic liver disease. Liver Transpl Surg 1997;3:3438 .

44. Kalaitzakis E, Sadik R, Holst JJ, Ohman L, Björnsson E. Gut transit is associated with gastrointestinal symptoms and gut hormone profile in patients with cirrhosis. Clin Gastroenterol Hepatol 2009;7:346-352.

45. Chander Roland B, Garcia-Tsao G, Ciarleglio MM, Deng Y, Sheth A. Decompensated cirrhotics have slower intestinal transit times as compared with compensated cirrhotics and healthy controls. J Clin Gastroenterol 2013;47:888-893.

46. Chen CY, Lu CL, Chang FY, et al. Delayed gastrointestinal transit in patients with hepatocellular carcinoma. J Gastroenterol Hepatol 2002;17:1254-1259.

47. Chang CS, Chen GH, Lien HC, Yeh HZ. Small intestine dysmotility and bacterial overgrowth in cirrhotic patients with spontaneous bacterial peritonitis. Hepatology 1998;28:11871190.

48. Nagasako CK, de Oliveira Figueiredo MJ, de Souza Almeida $\mathrm{JR}$, et al. Investigation of autonomic function and orocecal transit time in patients with nonalcoholic cirrhosis and the potential influence of these factors on disease outcome. J Clin Gastroenterol 2009;43:884-889.

49. Thalheimer U, De Iorio F, Capra F, et al. Altered intestinal function precedes the appearance of bacterial DNA in serum and ascites in patients with cirrhosis: a pilot study. Eur J Gastroenterol Hepatol 2010;22:1228-1234.

50. Chacko A. Colonic function in cirrhosis of liver \& in healthy controls. Indian J Med Res 1997;105:220-225.

51. Palma P, Mihaljevic N, Hasenberg T, Keese M, Koeppel TA. Intestinal barrier dysfunction in developing liver cirrhosis: an in vivo analysis of bacterial translocation. Hepatol Res 2007;37:6-12

52. Artis D. Epithelial-cell recognition of commensal bacteria and maintenance of immune homeostasis in the gut. Nat Rev Immunol 2008;8:411-420.

53. Bäckhed F, Ley RE, Sonnenburg JL, Peterson DA, Gordon JI. Host-bacterial mutualism in the human intestine. Science 2005;307:1915-1920.

54. McCormack TT, Sims J, Eyre-Brook I, et al. Gastric lesions in portal hypertension: inflammatory gastritis or congestive gastropathy? Gut 1985;26:1226-1232.

55. Chen LS, Lin HC, Lee FY, Hou MC, Lee SD. Portal hypertensive colopathy in patients with cirrhosis. Scand J Gastroenterol 1996;31:490-494.

56. Pienkowski P, Payen JL, Calès P, et al. Functional study, in man, of congestive gastropathy in cirrhosis by measurement of potential difference. Gastroenterol Clin Biol 1989;13:763- 
768.

57. Guslandi M, Foppa L, Sorghi M, Pellegrini A, Fanti L, Tittobello A. Breakdown of mucosal defences in congestive gastropathy in cirrhotics. Liver 1992;12:303-305.

58. Guslandi M, Sorghi M, Foppa L, Tittobello A. Assessment of mucosal defenses in portal hypertensive gastropathy. J Hepatol 1992;16:248.

59. Fukui H. Increased intestinal permeability and decreased barrier function: does it really influence the risk of inflammation? Inflamm Intest Dis 2016;1:135-145.

60. Barakat M, Mostafa M, Mahran Z, Soliman AG. Portal hypertensive duodenopathy: clinical, endoscopic, and histopathologic profiles. Am J Gastroenterol 2007;102:2793-2802.

61. Misra V, Misra SP, Dwivedi M, Gupta SC. Histomorphometric study of portal hypertensive enteropathy. Am J Clin Pathol 1997;108:652-657.

62. Bhonchal S, Nain CK, Prasad KK, et al. Functional and morphological alterations in small intestine mucosa of chronic alcoholics. J Gastroenterol Hepatol 2008;23(7 Pt 2):e43-e48.

63. De Palma GD, Rega M, Masone S, et al. Mucosal abnormalities of the small bowel in patients with cirrhosis and portal hypertension: a capsule endoscopy study. Gastrointest Endosc 2005;62:529-534.

64. Canlas KR, Dobozi BM, Lin S, et al. Using capsule endoscopy to identify GI tract lesions in cirrhotic patients with portal hypertension and chronic anemia. J Clin Gastroenterol 2008;42:844-848.

65. Korthuis RJ, Pitts VH, Granger DN. Intestinal capillary filtration in experimental diabetes mellitus. Am J Physiol 1987;253(1 Pt 1):G20-G25.

66. Kalaitzakis E. Gastrointestinal dysfunction in liver cirrhosis. World J Gastroenterol 2014;20:14686-14695.

67. Scarpellini E, Valenza V, Gabrielli M, et al. Intestinal permeability in cirrhotic patients with and without spontaneous bacterial peritonitis: is the ring closed? Am J Gastroenterol 2010;105:323-327.

68. Campillo B, Pernet P, Bories PN, Richardet JP, Devanlay M, Aussel C. Intestinal permeability in liver cirrhosis: relationship with severe septic complications. Eur J Gastroenterol Hepatol 1999;11:755-759.

69. Kalaitzakis E, Johansson JE, Bjarnason I, Björnsson E. Intestinal permeability in cirrhotic patients with and without ascites. Scand J Gastroenterol 2006;41:326-330.

70. Ersöz G, Aydin A, Erdem S, Yüksel D, Akarca U, Kumanlioglu K. Intestinal permeability in liver cirrhosis. Eur J Gastroenterol Hepatol 1999;11:409-412.

71. Huglo D, De Botton S, Canva-Delcambre V, et al. Simultaneous determination of pulmonary and intestinal permeability in patients with alcoholic liver cirrhosis. Eur J Nucl Med 2001;28:1505-1511.

72. Zuckerman MJ, Menzies IS, Ho H, et al. Assessment of in- testinal permeability and absorption in cirrhotic patients with ascites using combined sugar probes. Dig Dis Sci 2004;49:621-626.

73. Wang L, Llorente C, Hartmann P, Yang AM, Chen P, Schnabl B. Methods to determine intestinal permeability and bacterial translocation during liver disease. J Immunol Methods 2015;421:44-53.

74. Pascual S, Such J, Esteban A, et al. Intestinal permeability is increased in patients with advanced cirrhosis. Hepatogastroenterology 2003;50:1482-1486.

75. Dastych M, Dastych M Jr, Novotná H, Cíhalová J. Lactulose/ mannitol test and specificity, sensitivity, and area under curve of intestinal permeability parameters in patients with liver cirrhosis and Crohn's disease. Dig Dis Sci 2008;53:27892792.

76. Perera L, Shao L, Patel A, et al. Expression of nonclassical class I molecules by intestinal epithelial cells. Inflamm Bowel Dis 2007;13:298-307.

77. DeMeo MT, Mutlu EA, Keshavarzian A, Tobin MC. Intestinal permeation and gastrointestinal disease. J Clin Gastroenterol 2002;34:385-396.

78. Hossain MI, Nahar B, Hamadani JD, Ahmed T, Roy AK, Brown KH. Intestinal mucosal permeability of severely underweight and nonmalnourished Bangladeshi children and effects of nutritional rehabilitation. J Pediatr Gastroenterol Nutr 2010;51:638-644.

79. Bjarnason I. Intestinal permeability. Gut 1994;35(1 Suppl):S18-S22.

80. Bjarnason I, MacPherson A, Hollander D. Intestinal permeability: an overview. Gastroenterology 1995;108:1566-1581.

81. Keshavarzian A, Holmes EW, Patel M, Iber F, Fields JZ, Pethkar S. Leaky gut in alcoholic cirrhosis: a possible mechanism for alcohol-induced liver damage. Am J Gastroenterol 1999;94:200-207.

82. Pijls KE, Koek GH, Elamin EE, de Vries H, Masclee AA, Jonkers DM. Large intestine permeability is increased in patients with compensated liver cirrhosis. Am J Physiol Gastrointest Liver Physiol 2014;306:G147-S153.

83. Parlesak A, Schäfer C, Schütz T, Bode JC, Bode C. Increased intestinal permeability to macromolecules and endotoxemia in patients with chronic alcohol abuse in different stages of alcohol-induced liver disease. J Hepatol 2000;32:742-747.

84. Lee S, Son SC, Han MJ, et al. Increased intestinal macromolecular permeability and urine nitrite excretion associated with liver cirrhosis with ascites. World J Gastroenterol 2008;14:3884-3890.

85. Kim BI, Kim HJ, Park JH, et al. Increased intestinal permeability as a predictor of bacterial infections in patients with decompensated liver cirrhosis and hemorrhage. J Gastroenterol Hepatol 2011;26:550-557.

86. Othman M, Agüero R, Lin HC. Alterations in intestinal mi- 
crobial flora and human disease. Curr Opin Gastroenterol 2008;24:11-16.

87. Sekirov I, Russell SL, Antunes LC, Finlay BB. Gut microbiota in health and disease. Physiol Rev 2010;90:859-904.

88. Gupta A, Dhiman RK, Kumari S, et al. Role of small intestinal bacterial overgrowth and delayed gastrointestinal transit time in cirrhotic patients with minimal hepatic encephalopathy. J Hepatol 2010;53:849-855.

89. Bauer TM, Schwacha H, Steinbrückner B, et al. Small intestinal bacterial overgrowth in human cirrhosis is associated with systemic endotoxemia. Am J Gastroenterol 2002;97:2364-2370

90. Casafont Morencos F, de las Heras Castaño G, Martín Ramos L, López Arias MJ, Ledesma F, Pons Romero F. Small bowel bacterial overgrowth in patients with alcoholic cirrhosis. Dig Dis Sci 1996;41:552-556.

91. Wiest R, Albillos A, Trauner M, Bajaj JS, Jalan R. Targeting the gut-liver axis in liver disease. J Hepatol 2017;67:10841103.

92. Gómez-Hurtado I, Such J, Sanz Y, Francés R. Gut microbiota-related complications in cirrhosis. World J Gastroenterol
2014;20:15624-15631.

93. Chen Y, Yang F, Lu H, et al. Characterization of fecal microbial communities in patients with liver cirrhosis. Hepatology 2011;54:562-572.

94. Bajaj JS, Ridlon JM, Hylemon PB, et al. Linkage of gut microbiome with cognition in hepatic encephalopathy. Am J Physiol Gastrointest Liver Physiol 2012;302:G168-G175.

95. Liu H, Zhang S, Yu A, et al. Studies on intestinal permeability of cirrhotic patients by analysis lactulose and mannitol in urine with HPLC/RID/MS. Bioorg Med Chem Lett 2004;14:2339-2344.

96. Qin N, Yang F, Li A, et al. Alterations of the human gut microbiome in liver cirrhosis. Nature 2014;513:59-64.

97. Bajaj JS, Hylemon PB, Ridlon JM, et al. Colonic mucosal microbiome differs from stool microbiome in cirrhosis and hepatic encephalopathy and is linked to cognition and inflammation. Am J Physiol Gastrointest Liver Physiol 2012;303:G675-G685.son between patients with and without degenerative lumbar scoliosis. Eur Spine J. 2011;20(6):94753. 